\title{
Do the Laws of Nature Entail Causal Closure? Response to Michael Esfeld
}

\author{
Daniel von Wachter*
}

\section{Michael Esfeld's objection}

(1.1) $\mathrm{In}^{1}$ the article 'The Principle of the Causal Openness of the Physical' I claim that for an argument from the principle of the causal closure of the physical (PCC) for physicalism one needs a modal version of PCC: 'There cannot be a physical event that is not necessitated by preceding physical events.' Michael Esfeld objects that Kim's and Papineau's PCC should be understood as the non-modal claim that

For any physical event, insofar $p$ has a cause, it has a complete physical cause.

Therefore, Esfeld rightly suggests, 'causal completeness' would be a more suitable name than 'causal closure.' By a 'complete physical cause' Esfeld means not only a cause that includes all the partial causes and thus all the physical events that contribute to the causing, but one that includes all that is required for the causing, so that any additional causes would constitute causal overdetermination. But the term 'complete physical cause' neither implies that the cause necessitates the effect nor does it imply that the

1 This is a response to (Esfeld 2019).

* International Academy of Philosophy in the Principality of Liechtenstein

International Academy of Philosophy in the Principality of Liechtenstein, FürstFranz-Josef-Str. 19, FL-9493 Mauren, Liechtenstein

$\square$ epost@von-wachter.de W Website: http://von-wachter.de

(C) The Author. Journal compilation (C) The Editorial Board, Organon F. 
cause is non-probabilistic. Esfeld clarifies this by saying that 'PCC is not committed to determinism, since it leaves open to what extent physical events are caused.'

(1.2) In my article my response to the argument from non-modal PCC for physicalism was that the non-modal PCC can be justified only by evidence against the existence of non-physical objects. It therefore cannot be used in an argument for physicalism. Esfeld replies that the non-modal PCC can be known through the laws of nature: If the laws of nature are true, then nonmodal PCC is true, because the laws describe how the universe develops, and they refer only to physical forces as causes. Or rather, the laws describe a correlation between how the universe develops and physical forces. For example, according to determinism, "the propositions stating the laws of nature and the propositions describing the universe at an arbitrary time entail the propositions describing the state of the world at any other time.' If Esfeld's claim that the laws of nature entail PCC were true, then we could derive PCC from our knowledge of the laws. So the question before us is whether it is true that the laws of nature entail PCC.

(1.3) Esfeld's crucial assumption, which is shared by the majority of contemporary philosophers of science, is that laws of nature are differential equations that describe the evolution of physical systems, they describe what happens when. This entails the assumption that laws entail regularities of succession of the type 'All events of type $x$ are followed by events of type $y .{ }^{\prime 2}$ All the prominent philosophical theories of laws of nature share this assumption, they differ only in whether laws are more than regularities of succession and in what this 'more' is.

A 'fundamental and universal physical theory' describes the evolution of the whole universe. It would be a differential equation that links states of the physical universe at one time with states of the physical universe at other times. Let me call this the 'Comprehensive Differential Equation' $(\mathrm{CDE})$. If things always happen as descibed by this CDE, then the nonmodal PCC is true.

2 That this assumption is false is argued in (Wachter 2015). 
My response to Esfeld is that evolution equations are distinct from the laws of nature from which they are derived and that evolution equations require a no-further-causes clause while laws do not.

\section{Laws of nature are not differential equations}

(2.1) Laws of nature are different from the predictions and differential equations that we can derive from them. Let me first present the arguments by John Earman and John T. Roberts for this claim. They have argued that the fundamental laws do not contain ceteris paribus clauses, and that if a formula does contain ceteris paribus clauses, then it is not a fundamental law but part of a 'work-in-progress theory' (Earman and Roberts 1999; Earman, Roberts and Smith 2002).

'If laws are needed for some purpose, then we maintain that only laws will do, and if "ceteris paribus laws" are the only things on offer, then what is needed is better science, and no amount of logical analysis on the part of philosophers will render the "ceteris paribus laws" capable of doing the job of laws.' (Earman and Roberts 1999, 466)

(2.2) While they hold that laws do not require ceteris paribus clauses, they say that applications of a theory require what Carl Hempel called 'provisos.' As an example of an application of a theory, Hempel considers a description of the motion of two bodies that are 'subject to no influences from within or from outside the system that would affect their motions.' (Hempel 1988, 158) Earman and Roberts call such an application of a theory a 'differential equation of the evolution type' (Earman, Roberts and Smith 2002, 285); we can abbreviate this as 'evolution equation.' Discussing the proviso required for a system description, Hempel touches the issue of miracles:

The proviso must [...] imply the absence [...] of electric, magnetic, and frictional forces; of radiation pressure; and of any telekinetic, angelic, or diabolic influences. [(Hempel 1988, 158), also quoted in (Earman and Roberts 1999, 444)] 
So Hempel recognises the possibility of divine interventions, and he could have added interventions by souls. The proviso must state that the evolution equation does not apply to cases where there are such influences. To achieve this objective, he proposes the proviso, 'the total force acting on each of the two bodies equals the gravitational force exerted upon it by the other body; and the latter force is determined by the law of gravitation.' The expression 'total force' is supposed to exclude telekinetic, angelic, diabolic etc. influences. As a diabolic influence would be an action and, in my view, not a 'force' in the Newtonian sense, Hempel should say instead that 'nothing besides the gravitational force exerted by the two bodies is affecting their motion.' The point is clear: First, while laws of nature do not require ceteris paribus clauses or 'provisos,' evolution equations do. Secondly, ceteris paribus clauses are not enough, what is required is the nofurther-causes clause that implies that there are no other things acting on the objects.

(2.3) What then is a law of nature, in contrast to an evolution equation? According to Earman and Roberts, the law of gravitation, for example, asserts this:

(Regardless of what other forces may be acting) any two massive bodies exert a gravitational force on one another that is directly proportional to the product of their masses and inversely proportional to the square of the distance between them. (Earman and Roberts 1999, 473, footnote 14)

(2.4) So the evolution equations are not themselves properly called 'laws of nature.' Rather, $F=G \frac{m_{1} m_{2}}{d^{2}}$ is a laws of nature, and from this and the other laws one can derive differential equations that describe the development of systems in which nothing except the forces described by the laws used are affecting the movement of the two bodies. If there is a fundamental theory of physics, then perhaps a CDE can be derived from it, but CDE will not be the fundamental theory of physics itself, and it will describe the actual universe only if no non-physical objects are acting on it and only if no physical things have agent-causal powers.

[D]ifferential equations of evolution type are not laws; rather, they represent Hempel's applications of a theory to a specific case. 
They are derived using (unhedged) laws along with non-nomic modelling assumptions that fit (often only approximately) the specific case one is modelling. Because they depend on such nonnomic assumptions, they are not laws. For example, because Kepler's 'law' that planets travel in ellipses is derived from laws together with the assumption that there are only two bodies in the universe, it is not a law in spite of the normal nomenclature. [...] The 'law of free fall' is a consequence of a differential equation that involves the assumption that there is no resistance from the wind. That too is a non-nomic assumption, for it is not a law that there is no resistance from the wind. It seems to us that the role played by idealizations in physics is typically found here, in the derivation of differential equations, rather than within the laws themselves. (Earman, Roberts and Smith 2002, 286)

(2.5) Let me formulate Earman's and Roberts's point with my terminology. A law of mechanics says that there are forces of certain kinds in situations of certain kinds. More generally, using the notion of 'directedness' that I have introduced in the article (§ 4.14), A law of nature states that events of a certain kind are the bases of directednesses of a certain kind.

(2.6) Thus, contrary to Esfeld's view, a law of nature does not say that events of kind $x$ are always followed by events of type $y$. Laws do not entail regularities of succession. They speak not about what happens when but about Newtonian forces or, more generally, directednesses. As John Stuart Mill pointed out:

All laws of causation, in consequence of their liability to be counteracted, require to be stated in words affirmative of tendencies only, and not of actual results. (Mill 1843, book III, chap. $10, \S 5)$

(2.7) Evolution equations can be derived from laws by applying them to a particular system. From evolution equations we can derive statements of the form 'States of affairs of type $x$ on which nothing else is acting than the factors described in the equation are followed by events of type $y$.' Evolution equations describe the evolution of systems on which the factors that are taken into account in the equation and nothing else is acting. They require 
the no-further-causes clause. Laws of nature do not require such a clause because they describe, in the case of mechanics, forces, not actual movements, and the forces obtain under all circumstances (and in my view even necessarily) as the laws describe them.

\section{The universal physical theory}

(3.1) A comprehensive differential equation (CDE), which Esfeld calls the 'universal physical theory,' is an evolution equation that takes into account all the physical forces that exist. For the sake of the argument I grant that a CDE that can be applied to a state of the universe might exist, although the fact that we cannot even find a general closed-form solution for three-body systems raises doubts about this.

(3.2) Like all evolution equations, CDE would reqire the no-furthercauses clause 'if nothing else is acting on the objects.' One might object that a CDE will not require this, because it will take into account all factors. But first, CDE will not only be applicable to complete stages of the universe, but to all physical states of affairs. If it is applied to some state of affairs that is a part of the universe, then, because other things could affect the development of the system, the no-further-causes clause is required.

(3.3) If CDE is applied to a state of the whole universe, then there are still three ways how CDE could fail to apply. First, there could be physical forces that are not described by laws of nature. That is, there could be forces for which we cannot formulate a law that says that in situations of a certain type there are such forces. It is surprising that we can know any laws of nature and that we can make any predictions at all. Perhaps God created the universe so that all physical forces are governed by laws of nature, but it is a possibility that there are other physical forces, and the no-further-causes clause takes into account that possibility.

Second, perhaps there could be physical objects that can be agent causes. That is, they can by acting bring about choice events, i.e. events that are not the result of a law-governed causal process and thus have no preceding cause but occur through an action. A dualist will hold that choice events are brought about by souls, but somebody who holds the view that 
some material objects have consciousness can also hold that some material objects can bring about choice events.

Third, there could be a God or souls that sometimes cause physical events, in which case CDE does not apply. The data which justify CDE and the laws of nature justify only

- the proposition that there are the forces described by the laws, and

- the proposition that consists of CDE and the no-further-causes clause, as well as all other evolution equations with the no-furthercauses clause.

They do not justify the assumption that there are no non-physical things acting on the system. That is a metaphysical thesis that cannot be justified by physical experiments. Given my arguments for openness, it can only be justified by defeating the putative evidence for the various possible nonphysical objects. I conclude if we shall ever find a CDE, then its application will also require the no-further-causes clause und thus it will not entail PCC.

(3.4) CDE requires the no-further-causes clause for the same reason why all evolution equations require the no-further-causes clause: Physical causes are open to cooperation and to intervention, as I have argued in the article. If a physical state of affairs $S_{1}$ caused $S_{2}$, then something could have prevented $S_{2}$ so that $S_{1}$ would have occurred but not $S_{2}$. That this is true can be seen considering our experience of causal processes. Rolling billiard balls and tidal waves can be stopped. Also the process that is constituted by the complete physical universe is stoppable. Of course, if there are no nonphysical objects, then that process is never stopped. So would not CDE then be true also without the no-further-causes clause? Yes, but the proposition that CDE applies without the no-further-causes clause would not be justified by the experiments and observations through which we know the laws of nature.

(3.5) Let me clarify my position by commenting on some of Esfeld's statements. He writes: 'According to Newton, every change in the state of motion of a physical object is due to forces influencing the motion of the object.'

My response: This assumption entails that Newton's laws of motion exclude the existence of physical choice events and of physical events that 
have non-physical causes. Regardless of what Newton's view was, Newton's formulae can and should be used without this assumption. Newtonian physics describes which kinds of forces there are in which kinds of situations and says that if force $F$ and nothing else is acting on an object, then it accelerates with $a=F / m$. The assumption that there are no choice events and no physical events that have non-physical causes is not justified by the observations and experiments which justify Newton's laws.

(3.6) Esfeld denies that evolution equations require the no-further-causes clause: '[O]ne cannot add to this structure of physical theories formulated in terms of differential equations that indicate what determines the temporal evolution of the objects under consideration a clause to the effect that something may intervene from the outside that stops the evolution of the objects as indicated by what figures on the right hand side of the differential equation in question.'

My response: Evolution equations describe the development of a particular system only if no further forces are acting on the objects and only if no non-physical things are acting on the objects. Some might object that in order to do physics, we need to assume that all physical events are the results of causal processes and that we need no no-further-causes clause. This is what is called 'methodological naturalism.' But neither no-furthercauses clauses nor divine or human interventions would impede physics or violate the laws. ${ }^{3}$ To the contrary, predictions without the clause are not justified through the experiments and may turn out to be false. However, I agree that the fundamental physical theory is not an evolution description with a no-further-causes clause. It is not an evolution description or a differential equation at all, but a formula that is to be interpreted as saying that there are directednesses of certain kinds in situations of certain kinds. Like all laws of nature, it is true without no-further-causes clause and without ceteris paribus clause, and in my view, which I have not defended here, even necessarily. But it does not entail PCC.

(3.7) Esfeld suggests that 'the point at issue is [...] the metaphysics of laws.' 'One should be very cautious about using terms such as forces acting on objects. In physics, when objects interact, there is nothing that travels

3 More on this in (Wachter 2015). 
from one object to other objects and that literally pushes them to move in a certain manner. There is no justification to associate forces with agents that literally act on objects.'

My response: Does the view that there are no pushing forces - let us call it the Russellian view, because some have contested that Hume held it - fit better with the view that evolution equations require the no-further-causes clause or with the view that they do not? The denier of pushing forces cannot say, as I do, that laws are not evolution equations but describe that there are forces of certain kinds in situations of certain kinds. He has no plausible alternative to saying that laws are evolution equations. However, evolution equations with the no-further-causes clause lack the universality which we associate with laws. The no-further-causes clause destroys the entailment 'The propositions describing the initial condition of the system and the laws entail the propositions that describe the later states.' Therefore perhaps some deniers of pushing forces are drawn towards accepting evolution equations without the no-further causes clause.

On the other hand, I object to the Russellian and Humean view that the truth of evolution statements without the no-further-causes clause would be highly unlikely on the assumption that there are no pushing forces and no causal connections. In my view, the truth of the statement that includes the differential equations and the no-further-causes clause is evidence for the existence of pushing forces which can be counteracted and of directednesses. $^{4}$

(3.8) Esfeld's theory of laws is the majority view in philosophy of science. But why is it the majority view? What reasons do we have for believing it? Look at, for example, the law of gravitation, $F=G \frac{m_{1} m_{2}}{d^{2}}$, or at some more complicated law. I do not see anything there that suggests the majority view, because the formula says only something about forces. We can derive differential equations, but for systems that include less than the whole universe all agree that the differential equations describe only the evolution of those systems in which no further forces are acting than those taken into account in the equation. What reason do we have for believing that only

4 That laws explain regularities has been argued by Armstrong (1983, chap. 4) and by Foster (2004, chap. 3). 
forces and not agent causes can affect the system? Why should the differential equation that describes the universe not require the no-further-causes clause? And why should one think that the differential equations rather than the formulas from which they are derived are the laws and are in some sense more fundamental?

For the reasons given, I suggest that we should question the view that laws are evolution statements and entail regularities of succession and replace it by one that posits directednesses or, to use Mill's term, tendencies instead of regularities of succession. The laws, then, do not entail the principle of causal closure of the physical.

\section{References}

Armstrong, David M. 1983. What Is a Law of Nature? Cambridge: Cambridge University Press. https://doi.org/10.1017/CBO9781316499030

Earman, John, and John T. Roberts. 1999. "'Ceteris Paribus,' There Is No Problem of Provisos." Synthese 118 (3): 439-78. https://doi.org/10.1023/A:1005106917477

Earman, John, John T. Roberts, and Sheldon Smith. 2002. "Ceteris Paribus Lost." Erkenntnis 57 (3): 281-301. https://doi.org/10.1007/978-94-017-1009-1_1

Esfeld, Michael. 2019. "The Principle of Causal Completeness: Reply to Daniel von Wachter." Organon F 26 (1): 62-85. https://doi.org/10.31577/orgf.2019.26105

Foster, John. 2004. The Divine Lawmaker: Lectures on Induction, Laws of Nature, and the Existence of God. Oxford: Clarendon.

https://doi.org/10.1093/0199250596.001.0001

Hempel, Carl Gustav. 1988. "Provisos: A Problem Concerning the Inferential Function of Scientific Laws." Erkenntnis 28 (2): 147-64.

Mill, John Stuart. 1843. A System of Logic Ratiocinative and Inductive. New York: Harper, 1882.

von Wachter, Daniel. 2015. "Miracles Are Not Violations of the Laws of Nature Because the Laws Do Not Entail Regularities." European Journal for Philosophy of Religion 7 (4): 37-60. https://doi.org/10.24204/ejpr.v7i4.86 